\title{
Quantitative Radionuclide Assessment of Cardiac Dyssynchrony: Breakthrough in Patient Selection for Cardiac Resynchronization Therapy for Refractory Heart Failure?
}

\author{
Erica O. Miller ${ }^{1}$, Saurabh Malhotra ${ }^{2}$, and Ronald G. Schwartz ${ }^{1,3}$ \\ ${ }^{1}$ Cardiology Division, Department of Medicine, University of Rochester Medical Center Rochester, Rochester, New York; ${ }^{2}$ Division \\ of Cardiovascular Medicine, Jacobs School of Medicine and Biomedical Sciences, University at Buffalo, Buffalo, New York; \\ and ${ }^{3}$ Nuclear Medicine Division, Department of Imaging Sciences, University of Rochester Medical Center Rochester, Rochester, \\ New York
}

\begin{abstract}
A rently burden the human, medical, and financial resources of the United States. By 2030, this number is expected to increase to more than 8 million. In 2012, the total cost of HF in the United States was estimated at $\$ 30.7$ billion, which is expected to increase to $\$ 69.7$ billion by 2030 . HF contributes substantially to mortality and is documented on 1 in 9 death certificates in the United States (1). HF mortality $1 \mathrm{y}$ after diagnosis appears to be declining in the 21 st century according to Medicare data: $31.7 \%$ in 1999 to $29.6 \%$ in 2008 (2). These improvements have been attributed to evidence-based approaches in the management of $\mathrm{HF}$, including guideline-directed medical therapy, coronary revascularization, implantable cardioverter defibrillators, and cardiac resynchronization therapy (CRT) in which both the right and the left ventricles are paced synchronously to augment cardiac output (1).
\end{abstract}

\section{See page 1880}

With a reported number needed to treat of 24, CRT substantially reduces HF mortality at 1 y (3). The MIRACLE trial (2002) was the first double-blinded CRT trial and showed improvement in New York Heart Association (NYHA) functional class, quality of life, and ejection fraction as well as reduced HF hospitalizations in patients with $\mathrm{HF}$, left ventricular ejection fraction $\leq 35 \%$, and QRS duration $\geq 130 \mathrm{~ms}$ (4). Several landmark trials followed, including COMPANION, CARE-HF, RAFT, and MADIT-CRT, which demonstrated improvements in pathophysiologic HF indicators, HF admissions, and all-cause mortality (5-8). These trials included patients with markers of dyssynchrony such as prolonged QRS duration, with the CARE-HF trial requiring echocardiographic evidence of dyssynchrony for those with QRS duration 120-150 ms (6). So-

Received Jun. 4, 2016; revision accepted Sep. 21, 2016.

For correspondence or reprints contact: Ronald G. Schwartz, University of Rochester Medical Center, 601 Elmwood Ave., Box 679-N, Rochester, NY 14618.

E-mail: ronald_schwartz@urmc.rochester.edu

Published online Oct. 6, 2016.

COPYRIGHT (C) 2016 by the Society of Nuclear Medicine and Molecular Imaging, Inc.

DOI: $10.2967 /$ jnumed.116.177113 ciety guidelines accordingly began recommending CRT for select patients in 2008. The American College of Cardiology, the American Heart Association, and the Heart Rhythm Society currently recommend CRT for patients with left ventricular ejection fraction $\leq 35 \%$; NYHA class II, III, and ambulatory class IV symptoms; and evidence of dyssynchrony with QRS duration of $\geq 120 \mathrm{~ms}$ with stronger recommendation for $\mathrm{QRS}$ duration $\geq 150 \mathrm{~ms}$ and those with left bundle branch block (9-11).

Unfortunately, $30 \%-40 \%$ of CRT-treated patients are reported to have either no response or worsened HF. Limitations of patient selection technique and definition of CRT response likely contribute to high nonresponse rates (12-14). In this issue of The Journal of Nuclear Medicine, Badhwar et al. present a novel and effective approach to determining which patients most likely benefit from CRT using new measures of both intra-left-ventricular (LV) and interventricular dyssynchrony by planar equilibrium radionuclide angiography (ERNA) (15). This cardiac-blood-pool-based approach uses the first harmonic curve fit by phase and amplitude for each ERNA pixel as a surrogate for the timing and magnitude of regional contraction relative to the electrocardiograph (ECG) $R$ wave gating signal. In a previous report (16), ERNA-derived phase angle $(\varnothing)$ and amplitude quantitate regional contraction timing and magnitude and are the basis for synchrony and entropy parameters. Synchrony is the vector sum of all amplitudes based on the angular distribution of $\varnothing$ divided by the scalar sum of the length of all vectors, and entropy measures the disorder in the region of interest. These parameters were previously reported to be superior to SD of $\varnothing$ to distinguish hearts with normal, aneurysmal, diffuse dysfunction and severe regional dysfunction (16).

In the current study, ERNA measurements of LV synchrony (LVS) and interventricular synchrony (the difference between mean LVS and right-ventricular [RV] synchrony) were strongly predictive of response to CRT of NYHA symptom class. Lower baseline LVS and higher baseline interventricular synchrony predicted greater response to CRT with larger improvements in NYHA functional class. These findings are particularly relevant in patients with a QRS duration of 120-150 ms with left bundle branch block and those with QRS duration $\geq 150 \mathrm{~ms}$ with non-left bundle branch block morphology, in whom CRT is less strongly recommended.

Badhwar's planar ERNA approach has several advantages compared with other imaging techniques used to evaluate dyssynchrony and predict CRT response. In the past, numerous 
echocardiographic approaches have attempted to identify patients most likely to benefit from CRT. A major weakness of echocardiographic techniques is the lack of reproducibility. Although echocardiographic techniques assess dyssynchrony in either longitudinal or circumferential direction, the lack of a composite 3-dimensional echocardiographic assessment of ventricular dyssynchrony limits its effectiveness. Although 3-dimensional echocardiography reportedly enhances accuracy and reproducibility of the assessment of LV volumes and ejection frraction as well as assessment of myocardial dyssynchrony of the cardiac ultrasound approach $(17,18)$, its use in contemporary practice remains limited. Thus, an effective echocardiographic strategy for predicting CRT response remains elusive, and the American Society of Echocardiography recommends strongly against withholding CRT based on lack of echocardiographic evidence of dyssynchrony (19). Cardiac MRI is another strategy to evaluate dyssynchrony; however, widespread use is limited by cost, access issues, and incompatibility of many pacemaker and implantable cardioverterdefibrillator devices with MRI (20). By contrast, radionuclide imaging techniques such as planar ERNA can be safely performed in patients with pacemaker and implantable cardioverter-defibrillator devices and are highly reproducible and semiautomated, with limited operator intervention.

Radionuclide-based dyssynchrony assessment using phase analysis of gated SPECT myocardial perfusion image (MPI) data (21) has gained widespread recognition since its initial report of clinical utility for acute CRT response predictive of long-term HF outcome $(22,23)$. Both blood-pool and MPI techniques offer the advantage of excellent statistical sampling of up to hundreds of beats for quantitative assessment of dyssynchrony. The ERNA-blood-pool-based approach uses the first harmonic curve fit by phase and amplitude for each ERNA pixel as a surrogate for the timing and magnitude of regional contraction relative to the ECG $\mathrm{R}$ wave gating signal. As reported by Badhwar et al. in this issue of The Journal of Nuclear Medicine, however, ECG-gated SPECT MPI assessment of dyssynchrony relies on quantifying regional temporal differences in myocardial thickening of 600 myocardial voxels of myocardial count density during the summated cardiac cycle $(21,22)$. With this approach, acute change in synchrony before and after CRT with a single injection technique with ${ }^{99 \mathrm{~m}} \mathrm{Tc}$-sestamibi has been prospectively studied, and the highly predictive markers of improved short-term clinical outcomes include presence of baseline dyssynchrony, scar burden of $<40 \%$, and concordance of LV lead with the site of latest activation (22). The presence of baseline dyssynchrony on gated SPECT as a predictor of CRT response is similar to the predictive value of low LVS by planar ERNA noted in the current study. We now understand assessment of dyssynchrony alone as a predictor of CRT-response patients with medically refractory HF will be futile, as evidenced by echo-CRT (24). In addition to dyssynchrony, the major predictors of CRT response include scar burden and LV lead concordance with the site of latest activation $(25,26)$. The lack of assessment of these important determinants of CRT response from an ERNA-based approach appears disadvantageous. However, a limitation of SPECT MPIbased techniques for prediction of HF response to CRT includes attenuation and perfusion abnormalities that could result in undersampling of the myocardium, especially in the basal segments. The lack of dependency on geometric boundaries and the greater temporal resolution inherent to ERNA are advantages compared with MPI techniques for assessing dyssynchrony, especially interventricu- lar dyssynchrony, which is poorly assessed by SPECT because of a low RV count density. Additionally, ERNA is the most reproducible technique for assessment of the gatekeeper variable LV EF in patients with HF.

The incremental value of these radionuclide approaches to assess HR response to CRT of new measures of biventricular and interventricular dyssynchrony and the value of the acute response of these radionuclide indices of dyssynchrony by serial studies of ERNA or gated SPECT MPI are important hypotheses that will require large confirmatory clinical trials. Further research should include additional HF indicators such as LV EF; LV and RV volume indices by SPECT ERNA; metaiodobenzylguanidine neurohormonal activation $(27,28)$, on the 6-min walk; HF hospitalizations; the quality of life; and mortality.

A new window of opportunity confronts us as we grapple with the massive, looming clinical and economic burdens of HF in the 21 st century. Carefully planned large confirmatory clinical trials are warranted to evaluate the available evidence, which suggests quantitative radionuclide methods to measure cardiac dyssynchrony, and the acute quantitative responses to CRT with ERNA or ECG-gated SPECT MPI are effective strategies to predict longterm response of HF to CRT. Application of these techniques has the potential to prevent or limit futile CRT device implantations, expand access to CRT to those most likely to benefit, and improve the clinical and cost effectiveness of CRT for medically and surgically refractory $\mathrm{HF}$.

\section{REFERENCES}

1. Mozaffarian D, Benjamin EJ, Go AS, et al. Heart disease and stroke statistics: 2016 update-a report from the American Heart Association. Circulation. 2016; 133:e38-e360.

2. Chen J, Normand S-LT, Wang Y, Krumholz HM. National and regional trends in heart failure hospitalization and mortality rates for Medicare beneficiaries, 19982008. JAMA. 2011;306:1669-1678.

3. Fonarow GC, Yancy CW, Hernandez AF, et al. Potential impact of optimal implementation of evidence-based heart failure therapies on mortality. Am Heart J. 2011;161:1024-1030.

4. Abraham WT, Fisher WG, Smith AL, et al. Cardiac resynchronization in chronic heart failure. N Engl J Med. 2002;346:1845-1853.

5. Bristow MR, Saxon LA, Boehmer J, et al. Cardiac-resynchronization therapy with or without an implantable defibrillator in advanced chronic heart failure. $N$ Engl J Med. 2004;350:2140-2150.

6. Cleland JGF, Daubert J-C, Erdmann E, et al. The effect of cardiac resynchronization on morbidity and mortality in heart failure. $N$ Engl $J$ Med. 2005;352:1539-1549.

7. Tang ASL, Wells GA, Talajic M, et al. Cardiac-resynchronization therapy for mild-to-moderate heart failure. N Engl J Med. 2010;363:2385-2395.

8. Moss AJ, Hall WJ, Cannom DS, et al. Cardiac-resynchronization therapy for the prevention of heart-failure events. N Engl J Med. 2009;361:1329-1338.

9. Epstein AE, DiMarco JP, Ellenbogen KA, et al. ACC/AHA/HRS 2008 guidelines for device-based therapy of cardiac rhythm abnormalities: a report of the American College of Cardiology/American Heart Association Task Force on Practice Guidelines (Writing Committee to Revise the ACC/AHA/NASPE 2002 Guideline. J Am Coll Cardiol. 2008;51:e1-e62.

10. Tracy CM, Epstein AE, Darbar D, et al. 2012 ACCF/AHA/HRS focused update of the 2008 guidelines for device-based therapy of cardiac rhythm abnormalities: a report of the American College of Cardiology Foundation/American Heart Association Task Force on Practice Guidelines and the Heart Rhythm Society. Circulation. 2012;126:1784-1800.

11. Yancy CW, Jessup M, Bozkurt B, et al. 2013 ACCF/AHA guideline for the management of heart failure: a report of the American College of Cardiology Foundation/American Heart Association Task Force on practice guidelines. Circulation. 2013;128:e240-e327.

12. Ganjehei L, Razavi M, Massumi A. Cardiac resynchronization therapy: a decade of experience and the dilemma of nonresponders. Tex Heart Inst J. 2011;38:358-360. 
13. Fornwalt BK, Sprague WW, BeDell P, et al. Agreement is poor among current criteria used to define response to cardiac resynchronization therapy. Circulation. 2010;121:1985-1991.

14. Cleland J, Freemantle N, Ghio S, et al. Predicting the long-term effects of cardiac resynchronization therapy on mortality from baseline variables and the early response: a report from the CARE-HF (Cardiac Resynchronization in Heart Failure) Trial. J Am Coll Cardiol. 2008;52:438-445.

15. Badwhar N, James J, Hoffmayer KS, et al. Utility of equilibrium radionuclide angiogram-derived measures of dyssynchrony to predict outcomes in heart failure patients undergoing cardiac resynchronization therapy. J Nucl Med. 2016;2016:57:1880-1886.

16. O'Connell JW, Schreck C, Moles M, et al. A unique method by which to quantitate synchrony with equilibrium radionuclide angiography. J Nucl Cardiol. 2005;12:441450.

17. Thavendiranathan $\mathrm{P}$, Grant AD, Negishi $\mathrm{T}$, et al. Reproducibility of echocardiographic techniques for sequential assessment of left ventricular ejection fraction and volumes. J Am Coll Cardiol. 2013;61:77-84.

18. Russo C, Jaubert MP, Zin J, et al. Intra- and interobserver reproducibility of left ventricular mechanical dyssynchrony assessment by real time three-dimensional echocardiography. Echocardiography. 2012;29:598-607.

19. Gorcsan J, Abraham T, Agler DA, et al. Echocardiography for cardiac resynchronization therapy: recommendations for performance and reporting-a report from the American Society of Echocardiography Dyssynchrony Writing Group endorsed by the Heart Rhythm Society. J Am Soc Echocardiogr. 2008;21:191-213.

20. Chang SA, Chang HJ, Choi SI, et al. Usefulness of left ventricular dyssynchrony after acute myocardial infarction, assessed by a tagging magnetic resonance image derived metric, as a determinant of ventricular remodeling. Am J Cardiol. 2009;104:19-23.

21. Chen J, Henneman MM, Trimble MA, et al. Assessment of left ventricular mechanical dyssynchrony by phase analysis of ECG-gated SPECT myocardial perfusion imaging. J Nucl Cardiol. 2008;15:127-136.

22. Friehling M, Chen J, Saba S, et al. A prospective pilot study to evaluate the relationship between acute change in left ventricular synchrony after cardiac resynchronization therapy and patient outcome using a single-injection gated SPECT protocol. Circ Cardiovasc Imaging. 2011;4:532-539.

23. Chen J, Garcia EV, Bax JJ, Iskandrian AE, Borges-Neto S, Soman P. SPECT myocardial perfusion imaging for the assessment of left ventricular mechanical dyssynchrony. J Nucl Cardiol. 2011;18:685-694.

24. Ruschitzka F, Abraham WT, Singh JP, et al. Cardiac-resynchronization therapy in heart failure with a narrow QRS complex. N Engl J Med. 2013;369:1395-1405.

25. Adelstein EC, Tanaka H, Soman P, et al. Impact of scar burden by singlephoton emission computed tomography myocardial perfusion imaging on patient outcomes following cardiac resynchronization therapy. Eur Heart J. 2011;32:93103.

26. Saba S, Marek J, Schwartzman D, et al. Echocardiography-guided left ventricular lead placement for cardiac resynchronization therapy: results of the Speckle Tracking Assisted Resynchronization Therapy for Electrode Region trial. Circ Heart Fail. 2013;6:427-434.

27. Merlet $\mathrm{P}$, Valette H, Dubois-Randé J, et al. Prognostic value of cardiac metaiodobenzylguanidine in patients with heart failure. J Nucl Med. 1992;33:471-477.

28. Jacobson AF, Senior R, Cerqueira MD, et al. Myocardial iodine-123 metaiodobenzylguanidine imaging and cardiac events in heart failure. Results of the prospective ADMIRE-HF (AdreView Myocardial Imaging for Risk Evaluation in Heart Failure) study. J Am Coll Cardiol. 2010;55:2212-2221. 